\title{
The Tear-based Method for Preliminarily Diagnosing Diabetic Retinopathy at Home
}

\author{
Qin-Yi Chu \\ School of Life Science and Technology, ShanghaiTech University \\ chuqy@shanghaitech.edu.cn
}

\begin{abstract}
The purpose of the paper is to design a product for diabetic patient to preliminarily assess whether they suffer diabetic retinopathy at home. The whole design is about a cell-free paper-based test strips which can diagnose diabetic retinopathy (DR) even when it is in the first stage -- non-proliferative diabetic retinopathy (NPDR). When a tear dropped on it, the inflammatory cytokines it may contains combine to the corresponding inflammatory cytokines biosensor, then the catechol-2,3-dioxygenase $(\mathrm{C} 23 \mathrm{O})$ is translated. After that, drop colorless catechol on the test strips, it will turn into yellow in a few minutes. The degree of yellowing reflects the condition of patients. This test method can indirectly assess the intraocular cellular environment through tears to help doctors determine whether the patient had DR. It is simple and convenient, and has broad prospects for application in family medical care.
\end{abstract}

\section{CCS CONCEPTS}

- Applied computing $\rightarrow$ Life and medical sciences; Bioinformatics.

\section{KEYWORDS}

Diabetic retinopathy, Inflammatory cytokines, Test paper, Tear, Biosensor, Primary diagnosis

\section{ACM Reference Format:}

Qin-Yi Chu. 2021. The Tear-based Method for Preliminarily Diagnosing Diabetic Retinopathy at Home. In 2021 13th International Conference on Bioinformatics and Biomedical Technology (ICBBT '21), May 21-23, 2021, Xi'an, China. ACM, New York, NY, USA, 5 pages. https://doi.org/10.1145/ 3473258.3473298

\section{INTRODUCTION}

Diabetes is a common disease affects all ages, continents and communities in the world. In addition, diabetes is often accompanied by many complications, among which diabetic retinopathy (DR) is one of the most common. Studies have shown that $80 \%$ of people with diabetes for more than 20 years are affected by DR [1], and it is the leading cause of blindness in working-age adults in most developed countries [2].

Permission to make digital or hard copies of all or part of this work for personal or classroom use is granted without fee provided that copies are not made or distributed for profit or commercial advantage and that copies bear this notice and the full citation on the first page. Copyrights for components of this work owned by others than ACM must be honored. Abstracting with credit is permitted. To copy otherwise, or republish, to post on servers or to redistribute to lists, requires prior specific permission and/or a fee. Request permissions from permissions@acm.org.

ICBBT '21, May 21-23, 2021, Xi'an, China

(C) 2021 Association for Computing Machinery.

ACM ISBN 978-1-4503-8965-5/21/05 . .\$15.00

https://doi.org/10.1145/3473258.3473298
However, no warning signs might appear in the early stages of diabetic retinopathy. Patients may not notice they have NPDR unless they do fundus photography. As the condition progresses, patients are likely to have the following symptoms: spots or dark strings floating in vision (floaters), blurred vision, fluctuating vision, impaired color vision, dark or empty areas in your vision, vision loss, which can happen very rapidly [3, 4]. Moreover, though current treatments for diabetic retinopathy including laser photocoagulation, antiangiogenic therapy, and anti-inflammatory therapy are very effective in slowing down the disease and preventing further visual impairment [5], they cannot completely cure DR and may have some side effects [6]. The best way of delaying the progression of DR is to monitor it vigilantly and achieve optimal glycemic control [7]. Early diagnosis and early treatment for retinopathy can reduce the incidence of severe loss of vision in a high percentage of sight-threatening DR [2].

If a convenient and simple way of monitoring DR at home is developed, it will be good news for DR patients. Like thermometers, pregnancy testers and any other home diagnostic equipment, it allows patients easily understand their condition, so they can get timely diagnosis and treatment, or improve glycemic control to prevent rapid deterioration.

\section{PRELIMINARY THOUGHT AND OPERATION PROCESS}

No warning signs might appear in the early stages of diabetic retinopathy, so the purpose of the project is to design a product for diabetic patient to preliminarily assess whether they suffer diabetic retinopathy at home. The operation flow chart is shown in figure 1

The whole design is about a cell-free paper-based test strips which can diagnose diabetic retinopathy even when it is in the first stage - - non-proliferative diabetic retinopathy (NPDR). When a tear dropped on it, the inflammatory cytokines it may contains combine to the corresponding inflammatory cytokine's biosensor. Ideally, the complexes of inflammatory cytokines and biosensors themselves act as transcription operons/ translation factors, or the binding of those changes the conformation of regulatory proteins. Then, the catechol-2,3-dioxygenase (C23O) is translated. Alternatively, a modified ELISA kit which is more convenient for family use can be used to introduce catechol-2,3-dioxygenase (C23O) (Antibody be substituted by aptamer to lower the price). After that, drop colorless catechol on the test strips, it will turn into yellow in a few minutes. The degree of yellowing reflects the condition of patients.

The whole process is illustrated by figure 2 


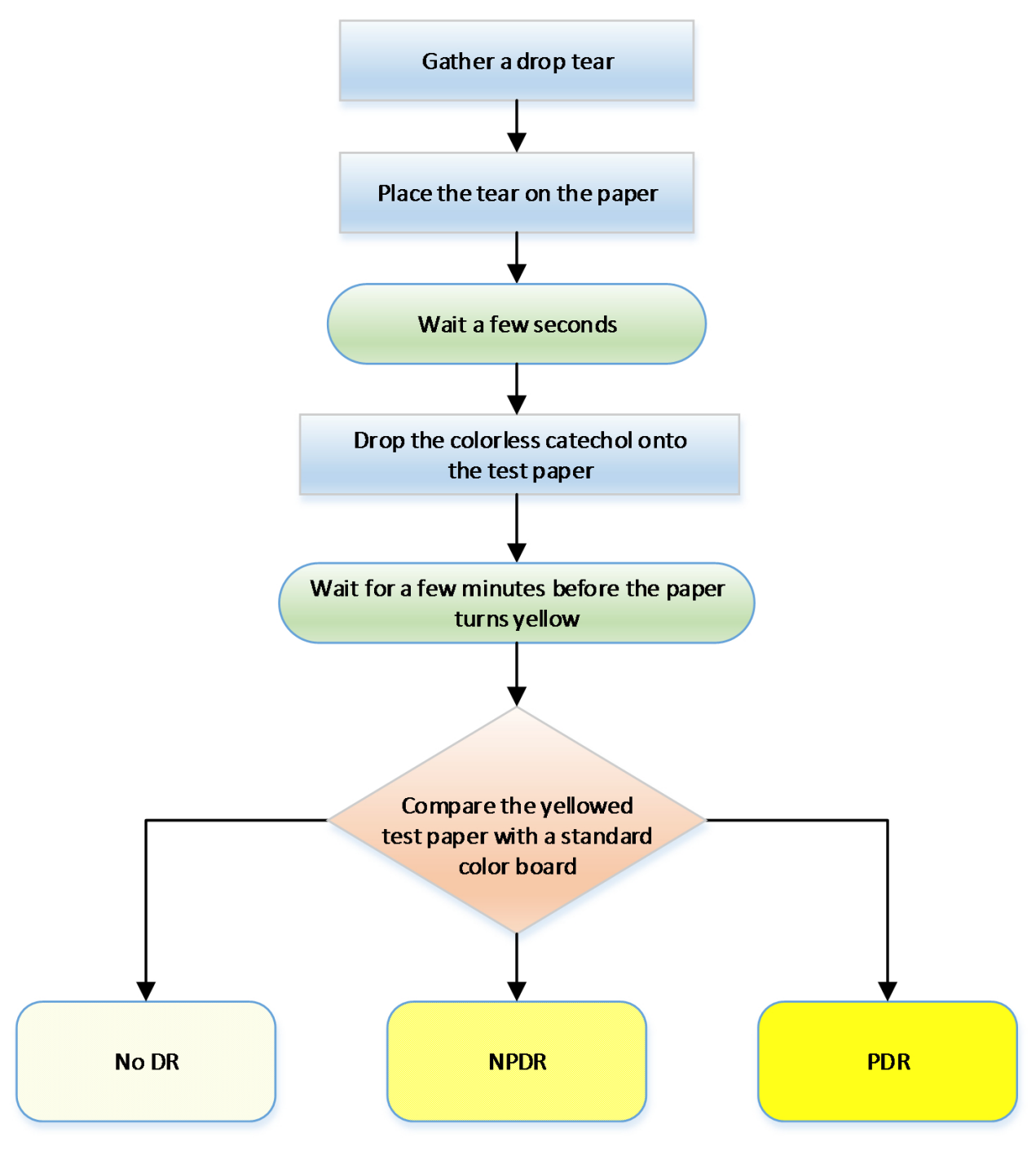

Figure 1: Operation flow chart

\section{RATIONALITY AND FEASIBILITY}

This project design conforms to scientific theory and research ethics that can be completed within one year based on the existing conditions.

\section{DESIGN THOUGHT}

\subsection{Why Use Tears?}

Tears are a complex biological mixture containing electrolytes, metabolites, lipids, mucins, some small organic molecules, and proteins. Different tear composition reflects different physiological condition of eye's underlying tissues. Therefore, the tear fluid can be used as a valuable source of biomarkers for objective analysis of ocular diseases. Moreover, the relatively high protein concentration of this fluid and the ease of noninvasive sample collection make it suitable for diagnostic and prognostic purposes [8].

As the purpose of the project is to let diabetic patient assess whether they suffer diabetic retinopathy at home, the material needed must be readily available and the detection method must be easy to operate. Tears are easily collected by the patient himself or herself, and compared to eye examination, tear-based diagnostic methods are hopeful to save complicated checking procedures, complex examining machines and medical professionals. As a result, tears are chosen to be the source of biomarkers for primary diagnosis of diabetic retinopathy at home in this project. 

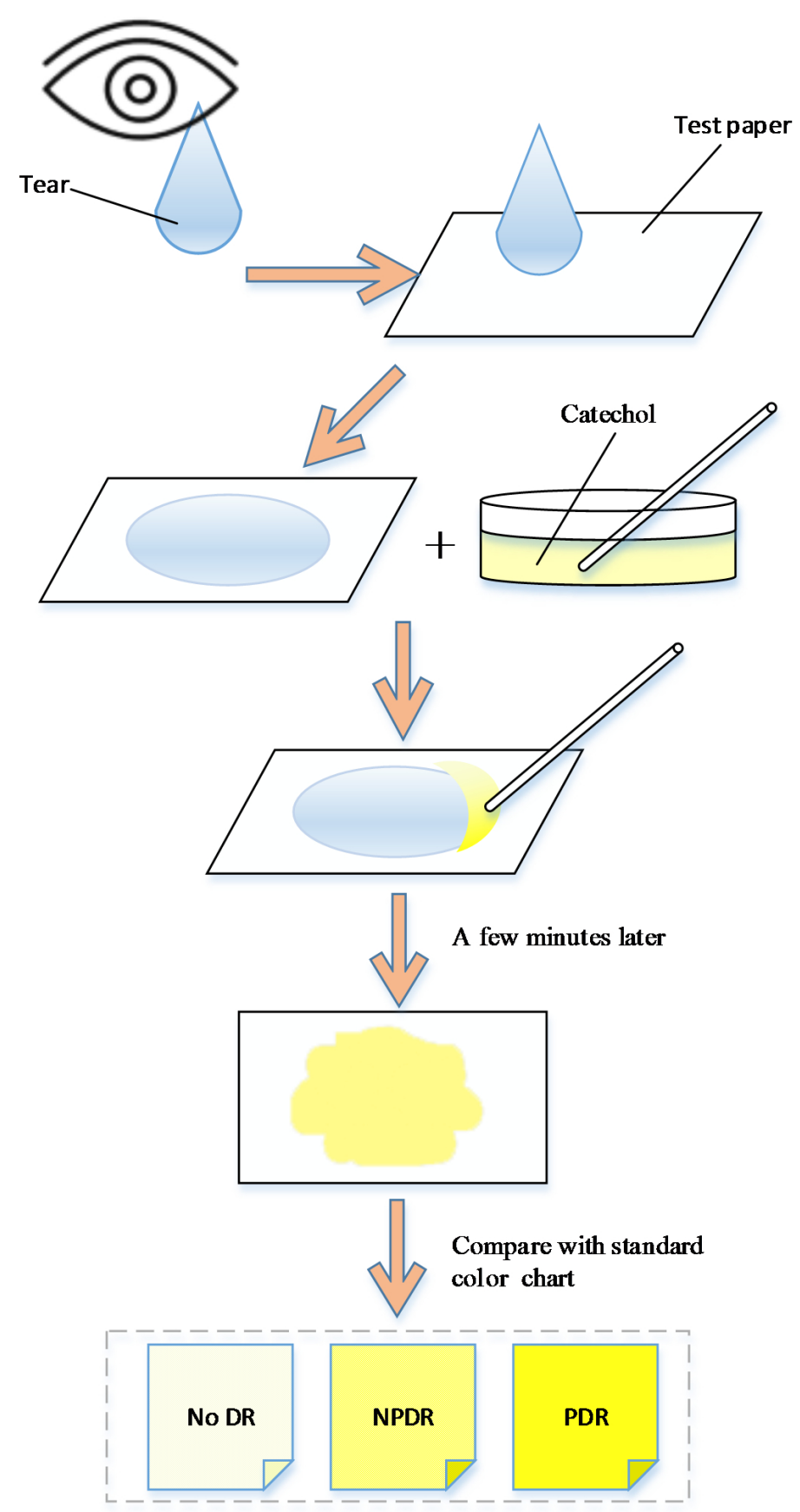

Figure 2: Determination process diagram.

\subsection{Why Choose Inflammatory Cytokines as Biomarkers?}

According to $\mathrm{Wu}$, Hwang, Song and Tao, aqueous concentrations of various cytokines increased with the severity of patients' diabetic retinopathy [9]. Their experiments found that inflammatory cytokines in the proliferative diabetic retinopathy (PDR) and NPDR groups were significantly higher than those in groups where people did not have diabetic retinopathy. In addition, the concentrations of IL-2, IL-5, and VEGF in aqueous humor were higher in the PDR group than those in the NPDR group. These conclusions indicate inflammatory cytokines like IL-2 and IL-5 can be used as biomarkers in diagnosis of diabetic retinopathy. 


\subsection{Why Use Cell-Free Paper-Based Test Strips?}

Due to the reason that the product of this project is expected to be extended to families affected by diabetes, it must be convenient to use and have comparatively low cost. Biosensors can potentially be produced at low costs [10]. Cell-free technique has the advantages of rapid response, high safety, long shelf life and flexibility for the addition or removal of natural or synthetic components [11-13], and paper-based test strips reduce costs further and portable [14] Therefore, cell-free paper-based test strips are used.

\subsection{Why Use C23O instead of GFP as a Signaling Protein?}

Catechol-2,3-dioxygenase (C23O) can cleave the substrate catechol into a colorful product, which can be observed as a change of color from transparent to yellow within a few minutes, visible by the naked eye $[15,16]$. However, the fluorescence of GFP is difficult to observe out of the lab compared to the change of color. Therefore, $\mathrm{C} 23 \mathrm{O}$ are used instead of GFP.

\section{NECESSARY EXPERIMENTS}

\subsection{Lab1 Detect Inflammatory Cytokines from Tears}

Purpose:To test if inflammatory cytokines could be detected in tears.

Procedure:

- Divide adult male Sprague-Dawley rats into healthy control group and diabetes group.

- For rats in diabetes group, establishing animal models by intraperitoneally injecting streptozotocin (STZ).

- Select appropriate number of NPDR rats and PDR rats, and label them as NPDR group and PDR group. Rats in diabetes group without DR form diabetes control group.

- Sample the aqueous humor of rats from each final group (healthy control group, diabetes control group, NPDR group and PDR group), and use cytometric bead array (CBA) technique to quantitatively detect inflammatory cytokines concentrated in aqueous humor, such as IL-2, IL-4, IL-5 and so on.

- Obtain reflex tears of rats from each final group by stimulation with mint oil or other things that can stimulate the production of tears, and collect them using glass capillary tubes [17].

- Use CBA to quantitatively detect inflammatory cytokines concentrated in aqueous humor, such as IL-2, IL-4, IL-5 and so on.

- Process and analyze data achieved, and judge whether enough amounts of inflammatory cytokines that can be used as biomarkers for DR is able to be detected from tears. Then, choose the most suitable one as biomarker.

\subsection{Lab2 Genetic Constructs Assembly}

Purpose: To assemble genetic constructs and test them. Procedure:

- Amplify plasmids in cultured E. coli KRX competent cells.
- Then, imported catechol-2,3-dioxygenase sequence xylE in them.

- Find applicable 5'-untranslated region(5'-UTR) and clone it between promoter and xylE by Gibson assembly to optimize C23O expression.

- Choose suitable biosensor for the biomarker selected in lab1, obtain its sequence, and clone it into the plasmid under control of the promoter and upstream of 5'-UTR- xylE.

- Amplify the gene of rraA, which is an endogenous E. coli RNase E inhibitor protein, via PCR from E. coli K12 genomic DNA.

- Clone rraA with N-terminal 6x-His-tag and TEV-protease cleavage site into the plasmids via Gibson Assembly.

- After that, remove unnecessary restriction sites via mutagenesis PCR.

- Use PCR or sequencing to verify whether the assembly is successful.

- Harvest the cells, disrupt via sonication, and centrifuge them.

- Add different concentrations of corresponding inflammatory cytokines and catechol into the mixture and observe the color change to test whether the plasmids can express in vitro [18].

[GFP can be used in lab to obtain precise and specific data and help model. Or methods such as spectrophotometry can be used to determine the color parameters of the $\mathrm{C} 23 \mathrm{O}$-contained solution and make model.]

\subsection{Lab3 Cell-Free Paper-Based Test Strips}

Purpose: To test whether cell-free paper-based test strips can detect inflammatory cytokines and thus diagnose DR, to make standard color chart

Procedure:

- Take some cell-free paper-based test strips and number each of them.

- Subsequently, obtain reflex tears of rats from each final group in lab1, drop them respectively onto different test strips, and note corresponding rat's condition.

- Add catechol onto each of the test strips and wait for them to change color.

- Observe whether the color of test strips getting deeper with the disease progresses and judge the effectiveness of them. If so, standard color chart can be made based on model.

[GFP can be used in lab to obtain precise and specific data and help model. Or methods such as spectrophotometry can be used to determine the color parameters of the $\mathrm{C} 23 \mathrm{O}$ test strips and make model.]

\section{CONCLUSION}

The novelty of the project is mainly reflected in the following three aspects:

- Diagnostic method: Current diagnostic method for DR are mostly based on the observation and analysis of eyes' structure and pathological changes or examination of a patient's 
eyesight, such as fundus photography, screening, visual acuity test and so on. Both of these ideas are completely abandoned by this design, which indirectly assessed the intraocular cellular environment through tears to help doctors determine whether the patient had DR.

- Technical means: Combine collecting tears, detecting inflammatory cytokines and paper-based test strips together, each of them is a novel and promising in the field of medical diagnosis. Moreover, C23O instead of more common GFP are used as a signaling protein, making the results easier to observe and distinguish.

- Household use: This is not a new concept in medical care, but it is a new attempt in diagnose DR. This method has the advantages of low cost, convenient operation and is suitable for all kinds of adults.

\section{REFERENCES}

[1] Kertes, P. J., \& Johnson, T. M. (Thomas M. (2007). Evidence-based eye care. Lippincott Williams \& Wilkins (LWW). Retrieved from https://www.ovid.com/productdetails.3534.html

[2] Valpuesta Martin, Y., Pacheco Callirgos, G. E., Maroto Martín, T. M., Piriz Veloso, M., Hernández Santamaría, S., \& López Gálvez, M. I. (2020). Satisfaction of patients and primary care professionals with a teleophthalmology-based screening programme for diabetic retinopathy in a rural area in Castilla y León, Spain. Rural and Remote Health, 20(1), 5180. https://doi.org/10.22605/RRH5180

[3] Diabetic Retinopathy: Definition and Patient Education. (n.d.). Retrieved January 23, 2020, from https://www.healthline.com/health/type-2-diabetes/retinopathy

[4] Diabetic retinopathy: Causes, symptoms, and treatments. (n.d.). Retrieved January 29, 2020, from https://www.medicalnewstoday.com/articles/183417.php

[5] Mitchell, P., \& Wong, T. Y. (2014). Management Paradigms for Diabetic Macular Edema. American Journal of Ophthalmology, 157(3), 505-513.e8. https://doi.org/ 10.1016/J.AJO.2013.11.012

[6] Wang, W., \& Lo, A. C. Y. (2018). Diabetic Retinopathy: Pathophysiology and Treatments. International Journal of Molecular Sciences, 19(6). https://doi.org/10. 3390/ijms19061816

[7] Hooper, P., Boucher, M. C., Cruess, A., Dawson, K. G., Delpero, W., Greve, M., . . Maberley, D. A. L. (2012). Canadian Ophthalmological Society evidence-based clinical practice guidelines for the management of diabetic retinopathy. Canadian
Journal of Ophthalmology. Journal Canadien d'ophtalmologie, 47(2 Suppl), S1-30, S31-54. https://doi.org/10.1016/j.jcjo.2011.12.025

[8] Azkargorta, M., Soria, J., Acera, A., Iloro, I., \& Elortza, F. (2017). Human tear proteomics and peptidomics in ophthalmology: Toward the translation of proteomic biomarkers into clinical practice. Journal of Proteomics, 150, 359-367. https://doi.org/10.1016/J.JPROT.2016.05.006

[9] Wu, H., Hwang, D. K., Song, X., \& Tao, Y. (2017). Association between Aqueous Cytokines and Diabetic Retinopathy Stage. Journal of Ophthalmology, 2017, 9402198. https://doi.org/10.1155/2017/9402198

[10] Kaur, H., Kumar, R., Babu, J. N., \& Mittal, S. (2015). Advances in arsenic biosensor development-a comprehensive review. Biosensors \& Bioelectronics, 63, 533-545. https://doi.org/10.1016/j.bios.2014.08.003

[11] Perez, J. G., Stark, J. C., \& Jewett, M. C. (2016). Cell-Free Synthetic Biology: Engineering Beyond the Cell. Cold Spring Harbor Perspectives in Biology, 8(12). https://doi.org/10.1101/cshperspect.a023853

[12] Pardee, K., Green, A. A., Takahashi, M. K., Braff, D., Lambert, G., Lee, J. W., . . . Collins, J. J. (2016). Rapid, Low-Cost Detection of Zika Virus Using Programmable Biomolecular Components. Cell, 165(5), 1255-1266. https://doi.org/10.1016/j.cell. 2016.04.059

[13] Pellinen, T., Huovinen, T., \& Karp, M. (2004). A cell-free biosensor for the detection of transcriptional inducers using firefly luciferase as a reporter. Analytical Biochemistry, 330(1), 52-57. https://doi.org/10.1016/j.ab.2004.03.064

[14] Hu, S.-W., Qiao, S., Pan, J.-B., Kang, B., Xu, J.-J., \& Chen, H.-Y. (2018). A paperbased SERS test strip for quantitative detection of Mucin-1 in whole blood. Talanta, 179, 9-14. https://doi.org/10.1016/j.talanta.2017.10.038

[15] Ingram, C., Brawner, M., Youngman, P., \& Westpheling, J. (1989). xylE functions as an efficient reporter gene in Streptomyces spp.: use for the study of galP1, a catabolite-controlled promoter. Journal of Bacteriology, 171(12), 6617-6624. https://doi.org/10.1128/jb.171.12.6617-6624.1989

[16] Zukowski, M. M., Gaffney, D. F., Speck, D., Kauffmann, M., Findeli, A., Wisecup, A., \& Lecocq, J. P. (1983). Chromogenic identification of genetic regulatory signals in Bacillus subtilis based on expression of a cloned Pseudomonas gene. Proceedings of the National Academy of Sciences of the United States of America, 80(4), 1101-1105. https://doi.org/10.1073/pnas.80.4.1101

[17] Schmut, O., Horwath-Winter, J., Zenker, A., \& Trummer, G. (2002). The effect of sample treatment on separation profiles of tear fluid proteins: qualitative and semi-quantitative protein determination by an automated analysis system. Graefe's Archive for Clinical and Experimental Ophthalmology = Albrecht von Graefes Archiv Fur Klinische Und Experimentelle Ophthalmologie, 240(11), 900905. https://doi.org/10.1007/s00417-002-0537-0

[18] Gräwe, A., Dreyer, A., Vornholt, T., Barteczko, U., Buchholz, L., Drews, G., . . Kalinowski, J. (2019). A paper-based, cell-free biosensor system for the detection of heavy metals and date rape drugs. PLOS ONE, 14(3), e0210940. https://doi.org/ 10.1371/journal.pone. 0210940 